\title{
Crowdsourcing como fuente de creatividad en el medio publicitario ecuatoriano
}

Crowdsourcing as a source of creativity in the Ecuadorian advertising industry

Natalie Barragán Castañeda Facultad de Comunicación de la Universidad de Especialidades Espiritu Santo, Ecuador.

E-mail: nbarraganconsultor@gmail.com

José Daniel Santibáñez Vásquez

Escuela Superior Politécnica del Litoral,

Ecuador.

E-mail: jdsantibanez@gmail.com

\author{
Ana Cristina Patiño Loor \\ Universidad de Especialidades Espíritu \\ Santo, Ecuador. \\ E-mail: anapatinoloor@gmail.com
}

Fecha de recepción: 06/04/2016 Fecha de aceptación: 30/09/2016
Palabras clave

- crowdsourcing

- marketing

- inteligencia colectiva

- medios sociales

- creatividad

\section{Resumen}

Crowdsourcing es un término acuñado por Jeff Howe en 2006, en la revista Wired, para referirse a un modelo de producción y de solución de problemas para las marcas. Este artículo da una introducción a las situaciones que enfrentan las empresas al momento de solucionar un problema 0 de buscar ideas creativas. En ese camino se ha desarrollado la técnica del Crowdsourcing convirtiéndose en una fuente valiosa de creatividad.

El presente documento también da a conocer las bases teóricas mediante la exposición de diversos autores que revelan sus puntos de vista sobre esta técnica, así como la explicación de casos internacionales y ecuatorianos que demuestran la eficacia del Crowdsourcing. En la investigación descriptiva se explora el conocimiento que tienen las empresas de Ecuador, principalmente de Guayaquil, en cuanto al Crowdsourcing y las oportunidades de utilizarla como una fuente de creatividad para las agencias de publicidad y áreas de mercadeo.

Finalmente, se concluye que existe un cierto conocimiento sobre Crowdsourcing, aunque en el medio publicitario ecuatoriano todavía no se dan pasos firmes respecto de la incorporación activa de los consumidores en los procesos creativos de la marca. 
Keywords

- crowdsourcing

- marketing

- collective intelligence

- social media

- creativity.

\begin{abstract}
Crowdsourcing is a term coined by Jeff Howe in 2006 in Wired magazine, referring to a production and troubleshooting model for brands. This article provides an introduction to situations faced by different brands and companies when solving a problem or looking for creative ideas. In the same vein, the Crowdsourcing technique was developed and had become a valuable source of creativity.

This document also discloses the theoretical basis by exposing various authors who reveal their views on this technique, as well as the explanation of international and Ecuadorian cases demonstrating the effectiveness of Crowdsourcing. In the descriptive research, knowledge on Crowdsourcing by Ecuadorian companies, especially from Guayaquil, is explored in terms of opportunities as a source of creativity for advertising agencies and marketing departments.

Finally, it is concluded that there is some knowledge about Crowdsourcing but the Ecuadorian advertising field has not yet given firm steps regarding the active involvement of consumers in brand creative processes.
\end{abstract}

\section{Introducción}

En las últimas décadas, la creatividad para el marketing y la comunicación ha pasado por importantes cambios, ya que las técnicas utilizadas para vender 0 comunicar un producto o servicio no son las mismas de años atrás. Al tener los consumidores fácil acceso a la información mediante los avances tecnológicos, las empresas necesitan reconsiderar sus acciones al momento de diseñar estrategias y publicidad. El networking, la red de contactos profesionales en medios sociales, lleva a las marcas a buscar nuevas ideas que les permita adaptarse a las actuales preferencias y necesidades de comunicación de los consumidores (Galmés, 2012). Por otro lado, los consumidores han pasado de estar totalmente aislados a estar conectados con la marca; de estar desinformados a estar totalmente informados y, por último, han pasado de una actitud pasiva a una actitud activa, todo gracias a los avances tecnológicos.

Estos antecedentes llevan a las empresas a reconsiderar formas diferentes para obtener resultados, nuevos insights (aspectos contenidos en la mente del consumidor, percepciones) y, sobre todo, para complacer a sus consumidores. Es en esta problemática donde la técnica del Crowdsourcing entra como fuente de creatividad para dar una respuesta a las necesidades de las marcas. El Crowdsourcing surgió como una forma de externalizar trabajos y de aprovechar las mejores ideas de un colectivo en internet, pero, gracias a su éxito, se ha transformado en un modelo de negocios en el que ya confían muchas empresas de diferentes sectores de actividad (El confidencial, 2011). El presente estudio plantea como propósito explicar en qué consiste el Crowdsourcing e identificar las oportunidades de utilizar esta técnica como una fuente de creatividad y de resolución de problemas para el área de mercadeo y de las agencias de publicidad en el mercado ecuatoriano. Las preguntas que se pretenden responder son:

a) ¿Qué es Crowdsourcing?

b) ¿Qué tan difundida está la técnica del Crowdsourcing en nuestro medio?

c) ¿Cuáles son las ventajas percibidas del uso del Crowdsourcing como fuente de creatividad de las agencias de publicidad y áreas de mercadeo? 
d) ¿En qué aspectos se requiere trabajar localmente para que esta técnica se desarrolle y se utilice con mayor frecuencia?

Para dar respuesta a estos interrogantes se abordará, en primer lugar, los conceptos y proposiciones que desde el marco teórico explican el Crowdsourcing y su relación con el marketing. Luego se explicará, a través de casos reales, su aplicación, tanto en mercados y marcas globales como del Ecuador. Finalmente, se tomarán fuentes primarias a través de encuestas a profesionales del sector de la comunicación y los negocios, de modo que sean ellos quienes expongan su visión de cómo el uso de esta técnica de marketing puede influenciar en la generación de propuestas publicitarias y comerciales.

\section{Marco teórico}

Jeff Howe (2006), en la revista Wired, describe por primera vez el término Crowdsourcing como un fenómeno en el que cada día las personas utilizan su tiempo libre para ayudar a resolver problemas de gran importancia. Además, define al Crowdsourcing con un concepto que está compuesto por dos partes. Crowdsourcing es el acto de tomar una actividad llevada a cabo tradicionalmente por un agente designado previamente, y externalizarlo en un grupo de personas indefinido, en forma de convocatoria abierta, mediante la aplicación de los principios del software libre a otros campos. Utilizando la técnica del Crowdsourcing, las empresas buscan ideas creativas, soluciones o respuestas, y también delegan ciertas tareas importantes a un grupo diverso y descentralizado de networkers. Según Piller y Walcher (2006), el Crowdsourcing se basa en un sistema de autoselección en donde los usuarios finales deciden por sí mismos si participan o no en el proceso. Por lo tanto, establecen que la técnica del Crowdsourcing depende mucho del usuario final y de su disposición por resolver problemas y dar ideas creativas.
Por otra parte, Brabham (2008) coincide con la definición de Howe, ya que él afirma que la mayoría de las iniciativas del Crowdsourcing se utiliza para resolver problemas y/0 diseñar productos 0 servicios, en beneficio de las compañías, en ocasiones por medio de no expertos y principiantes.

James Surowiecki lo plantea de otra forma basándose en su teoría de que un grupo, en las circunstancias correctas, es más inteligente que el miembro más inteligente del grupo, es decir, que las decisiones tomadas por una gran cantidad de personas siempre se acercará más a la decisión correcta que las decisiones tomadas individualmente (Shuurman, Baccarne, De Marez y Mechant, 2012). Además, en el mismo estudio se refiere al concepto de Crowdsourcing y habla de un término importante denominado la sabiduría de las masas. Este autor menciona que, para que funcione la llamada sabiduría de las masas, algunas condiciones deben cumplirse, entre ellas: diversidad de opiniones, independencia en opiniones, descentralización del conocimiento y agregación a un pensamiento colectivo. Estas cuatro condiciones deben darse siempre y cuando estén basadas en la masa o el Crowd.

Por último, Andrea Grover (2006), reconocida artista, escritora y también promotora de arte basado en Crowdsourcing, coincide en que deben cumplirse dos condiciones: la independencia en las opiniones y la descentralización de conocimiento, ya que los individuos tienden a ser más abiertos y a mostrar menos limitaciones cuando no están siendo juzgados o examinados, físicamente.

\section{Características del Crowdsourcing}

En primer lugar, se utiliza una palabra clave: amateurismo. La conexión de este término con el tema de estudio se puede observar en el momento en que la empresa pide a una masa de gente que se puede denominar «principiantes» para que ayuden en la búsqueda de una solución (Roig, SanchezNavarri y Leibovitz, 2012). 
En segundo lugar, se encuentra el concepto de Open source, como un modelo de software libre. La explosión de la utilización de las fuentes abiertas hizo que la técnica sea más factible, ya que al unir el conocimiento del colectivo con esta fuente, se podía lograr resultados positivos.

En tercer lugar, se encuentra el fácil acceso de las personas para utilizar las herramientas de producción. Es decir, para que la técnica se haya desarrollado, un requisito indispensable era que el colectivo tuviera una capacidad de acceso rápido y frecuente para producir un contenido y a la vez dar una solución.

En cuarto lugar, Howe (2006) hace alusión a un término importante: la autogestión. Para el funcionamiento del Crowdsourcing, el crecimiento de las empresas 0 entidades con la capacidad de autogestionarse y de buscar una meta en común es fundamental.

\section{Relación entre Marketing y Crowdsourcing}

En la actualidad, son muchas las áreas de marketing y publicidad que hacen uso del Crowdsourcing para obtener ideas creativas y solucionar problemas para sus marcas; y esto lo hacen a través de las cuatro estrategias dadas por Howe, entre ellas:

- el Crowdfunding (proyecto creado para recaudar fondos)

- el Crowdcreation (individuos que dan soluciones a un problema específico)

- el Crowdvoting (los individuos votan por la mejor opción)

- otros tipos de Crowdsourcing.

La importancia de mencionar estas cuatro estrategias radica en el valor que tiene cada una de ellas en el marketing y el aporte que dan, dependiendo de los objetivos que se quiere lograr.

Hoy en día, por ejemplo, la más utilizada y explotada es el crowdcreation. Kotler (2014), en su libro 8 maneras de crecer, expone que las empresas ya no se limitan a ver a los consumidores como seres pasivos sino, más bien, los invitan a participar en la creación de nuevas ideas. La cocreación es una estrategia especialmente útil en los mercados $B 2 B$--negocios dirigidos de empresas a empresas (Business to Business)-, ya que en estos negocios la conexión con el cliente es vital. Kotler dice: la innovación requiere un cierto grado de coordinación entre el innovador y su público objetivo.

Entre los grandes ejemplos de cocreación que encontramos en esta obra se encuentran marcas líderes como Harley-Davidson y LEGO.

Harley-Davidson es un ejemplo perfecto de lo que menciona Kotler, pues la marca ha llegado a coordinarse de tal manera con sus consumidores que muchos de ellos, expertos en mecánica, piden visitar las instalaciones para poder ayudar a los ingenieros de Harley-Davidson a encontrar soluciones a sus problemas técnicos, sin querer ninguna remuneración a cambio.

Por otro lado, LEGO siempre está en constante búsqueda de nuevas estructuras que puedan construirse con su juego, por lo que la empresa invita a jóvenes, a través de su página web, a que den ideas y sugieran nuevas formas para dichas estructuras.

La Fiat continúa mejorando sus modelos cada año gracias a la colaboración de sus consumidores. El Fiat Mio fue creado en base a un concurso en donde la empresa de automóviles invitó a personas de todo el mundo a enviar ideas sobre un coche futurista.

Estos ejemplos son sólo un vistazo general sobre la utilización de esta técnica y de la eficacia que ha tenido alrededor del mundo. Para enfocar el tema de estudio a un ejemplo específico es importante conocer las fases que hacen que se desarrolle la técnica y las figuras que intervienen en la misma.

\section{Fases del Crowdsourcing}

Como todo proceso, el Crowdsourcing está determinado por varias fases que permiten que la técnica se utilice correctamente y dé los resultados buscados. 
Por este motivo, es importante conocer primero, de manera general, las cuatro figuras esenciales que intervienen en este proceso:

- el medio o la plataforma de Crowdsourcing

- el Crowdsourcer, aquel que publica el concurso

- el Crowdworker, las personas o la comunidad que da una respuesta a los problemas de la empresa

- el Crowdmanager, la persona que se encarga de la gestión y de la interacción con los participantes para que éste se desarrolle correctamente (Figura 1).

Figura 1. Fases del Crowdsourcing

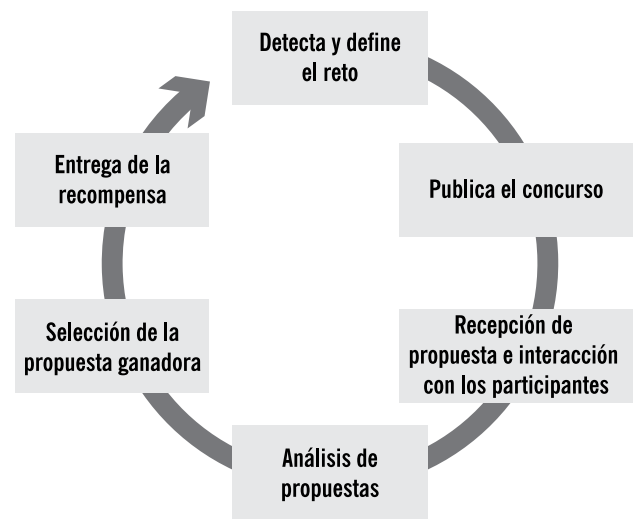

Fuente: Azcarate (2013)

1. Detecta y define el reto. Antes que nada, la marca debe estar segura de cuál es el problema que quiere resolver y definir con minuciosidad cada detalle que necesite que el crowd sepa sobre el problema, para evitar ambigüedades.

2. Publicación del concurso. La marca debe definir cuál es el medio por el que va a dar a conocer el concurso 0 , en su defecto, cuál plataforma se adecúa mejor a su necesidad y al problema que quiere resolver.

3. Recepción de propuestas e interacción con los participantes. La cantidad de información obtenida sólo va a ser útil si se la recepta de manera correcta. En esta fase es imprescindible tener un moderador que organice la información o las propuestas que van entregando los participantes (Azcarate, 2013). 4. Análisis de las propuestas. La marca analiza en profundidad y estudia las posibilidades de cada proyecto. El análisis no sólo debe ser del lado positivo, sino también del negativo, para poder evaluar si aquella propuesta va a tener repercusiones en la empresa.

5. Selección de la propuesta ganadora. Debe ir de la mano con un análisis profundo de todos los factores que intervienen en el proceso, ya que podría existir una muy buena propuesta y con grandes resultados, pero no estar bien planificada por parte del crowdsourcer.

6. Entrega de la recompensa. Es importante que la recompensa sea atractiva para la comunidad, ya que es uno de los mayores incentivos para que ésta le dé importancia a la solución que vaya a proponer.

Una vez que se ha desglosado el concepto y las bases que sustentan el Crowdsourcing, se da paso a la aplicación de este sistema en la acción comercial, a través de distintas de técnicas investigación.

\section{Marco Metodológico}

\subsection{Diseño de la Investigación}

Como herramientas de recolección de datos se utilizó el método exploratorio, descriptivo y documental. El método exploratorio acerca a una mejor definición del problema a investigar y se nutre principalmente de fuentes secundarias. Sin embargo, al no ser estructurada, sus datos no son concluyentes (Martínez Carrasco, 2011). El método descriptivo, según (Martinez Rodríguez, 2011), trabaja con las propias palabras de las personas y se lo hace mediante la observación de la propia conducta. Por otro lado, el método documental consiste en reunir, seleccionar y analizar datos producidos por la sociedad para un estudio determinado (Gómez, Grau, Ingellis y Jabbaz, 2010). Las fuentes de información utilizadas fueron documentos escritos, como revistas científicas, libros, bases académicas y periódicos. 
Adicionalmente, se realizaron encuestas a profesionales del medio publicitario y de mercadeo en el país para poder conocer su perspectiva del Crowdsourcing en el Ecuador y su experiencia con esta técnica. Este tipo de investigación está dentro del método descriptivo mencionado anteriormente.

El diseño parte de una lógica deductiva, ya que va de lo general a lo particular, pues se comienza investigando sobre las generalidades de la técnica y ejemplos internacionales, hasta llegar a la evaluación del Crowdsourcing en Ecuador, en base a las experiencias de las personas del medio.

\subsection{Análisis de la información}

La información se realizó en base a un análisis transversal ya que este tipo de estudio permite concentrarse en un determinado grupo de población (Shuttleworth, 2010). Como se mencionó anteriormente, las interrogantes que se pretende responder con esta investigación son: definir qué es Crowdsourcing, cuán difundida está la técnica en nuestro medio, las ventajas percibidas de su uso, y por último, las oportunidades que existen en el mercado ecuatoriano para que se desarrolle.

El primer interrogante abre paso al análisis de los ejemplos del Crowdsourcing en profundidad, ya que muchas veces, a través de los ejemplos, es como se puede demostrar la eficacia de las cosas. El análisis del ejemplo se lo realizará en base a las fases anteriormente descritas en el marco teórico y a los agentes esenciales que intervienen en estas fases.

\section{Caso Doritos}

En 2008, Doritos, una marca de tortilla chips sazonados, mundialmente reconocida, decidió que para ganar relevancia y fidelizar a sus consumidores debía conectarse con ellos de una manera especial y única. Para lograr esa fidelización, la empresa decidió poner al aire un concurso en donde las personas realizaran un spot publicitario de 30 segundos y que, como recompensa, éste iba a ser visto por millones de personas en el juego final del SuperBowl.

Los objetivos de esta campaña eran tres, según el brief expuesto por los Effie Awards (2008):

1. Conseguir un crecimiento en el volumen de ventas superior al $4 \%$ durante la campaña.

2. Se plantearon como meta recibir 200 presentaciones de comerciales para el concurso.

3. Lograr $\$ 5$ millones en retorno por Relaciones Públicas.

Para lograr estos objetivos, la empresa debía tener muy claro cuál era su público objetivo. Doritos, en sus estudios, encontró que su target eran jóvenes entre 16 y 24 años, que tenían como característica estar conectados, informados y, sobre todo, creaban cultura. Eran jóvenes que utilizaban lo digital como un utilitario en su vida. El $60 \%$ de su grupo objetivo había estado sumergido en el mundo de las redes sociales y estaban constantemente revisando blogs y páginas web que les diera información relevante y que generaran contenido.

Estos antecedentes dan paso a la primera fase: Se detecta y se define el reto. Doritos tenía claro cuál era el objetivo: ganar relevancia y fidelizar a sus consumidores. Definió, a su vez, de qué manera lo iba a realizar: mediante un concurso de spot publicitario. Además, tenía claro cuál era su público objetivo y cuáles detalles quería que el crowd supiera.

La segunda fase se desarrolló una vez que la marca tuvo claro el problema y el target. La marca invitó a los consumidores o crowdworkers a crear un spot publicitario de 30 segundos, en donde mostraran la pasión de consumir Doritos como un snack. Para llegar a las personas interesadas en participar en el concurso y sobre todo a los crowdworkers con experiencia en producción de videos, la marca adquirió dos programas que se utilizan para subir videos: MySpace Video HomePage y Music HomePage Roadblocks, además de hacer promoción de su concurso en lugares estratégicos de la red, donde sus consumidores normalmente navegaban, como Youtube 
y Yahoo, mediante el uso de banners digitales segmentados a los perfiles de usuarios de 16 a 24 años. Los participantes tenían cinco semanas para producir y subir el vídeo.

La fase tres, una de las fases más importantes, ya que aquí es donde se ubica la recepción de propuestas e interacción con los participantes, se da cuando la marca empieza a recibir los spots publicitarios. La manera en que la marca receptó la información fue a través de un programa llamado Yahoo Instantmessage. Adicionalmente, crearon una comunidad en MySpace donde las personas podían ver, votar y comentar sobre los videos que más le llamaban la atención. La comunidad que se creó alrededor de este concurso permitió a la marca establecer una gran relación con el consumidor pues tenían acceso a todas las opiniones de sus consumidores, así como crear un ambiente de fidelización hacia la marca y conectarse a través del ambiente viral de la comunidad.

La marca desarrolló la cuarta fase del Crowdsourcing al momento de analizar los spots publicitarios. Un comité de panelistas y expertos en el tema revisó cada uno de los vídeos tomando como parámetros la originalidad y la creatividad, lo asertivo de las propuestas y, por último, si iba o no dirigido al target. Estos panelistas, durante todo su proceso de revisión, llegaron a cinco finalistas.

La elección de estos finalistas permitió que se desarrollara la quinta fase del Crowdsourcing, ya que es en esta instancia donde se elige la propuesta que mejor se adhiera a sus objetivos de comunicación y de ventas.

Por último, en el paso más interesante para el crowdworker que es la premiación, el spot ganador fue premiado mediante la exposición de su video en uno de los eventos más importantes del mundo, el SuperBowl. ¿Cómo saber si estos pasos resultaron eficaces para el correcto desempeño de la campaña? Pues se lo midió en el cumplimiento de sus objetivos. El volumen de ventas que estimaban subir en un $4 \%$ subió realmente a un $12 \%$, siendo este un éxito para la empresa. En vez de recibir 200 comerciales, que era lo estimado, recibieron 1,071 spots publicitarios y, por último, los $\$ 5$ millones en relaciones públicas supusieron un éxito, ya que tuvieron 20 artículos inéditos en las primeras páginas de los periódicos, 1 billón de impresiones en medios y más.

Claro está que la marca, a través de la realización de esta actividad, cumplió con los objetivos que se había propuesto, pero detrás de todo este éxito «numérico», la marca se enriqueció con mucho más que 200 comerciales. Las verdaderas ventajas del Crowdsourcing salieron a relucir en esta actividad que permitió que la marca se conectara con sus consumidores, que recibiera feedback interno y permanente para campañas a futuro y que existiera una generación de ideas innovadoras dadas por un gran grupo de personas mucho más diverso que el pequeño grupo de publicistas ejecutivos con el que cuenta una agencia de publicidad tradicional y todo esto a un bajo costo (Whitla, 2009).

\section{Caso Batterycentros MAC}

En 2013, Batterycentros Mac buscaba posicionarse en el mercado joven como un producto social a través del primer comercial de la marca en Ecuador (Premios Iberoamericanos Social Media, 2013). La agencia de publicidad que llevaba la cuenta, Agencia Laika, decidió utilizar la técnica del Crowdsourcing para generar ideas para esta campaña.

El primer paso fue ingresar en las redes sociales y mostrar de manera creativa los peores escenarios en que un vehículo no arranca. Estos insights crearon un engagement (grado con el que los consumidores interactúan con la marca) con los consumidores de la marca, y estos comenzaron a compartir sus historias y, de esa manera, los fans se comenzaron a familiarizar más con la marca. Los resultados fueron tan exitosos y la información que obtuvo la agencia fue tan valiosa, que decidieron crear una campaña llamada El plan perfecto. 
Consistía en poner al aire un concurso de comerciales en los que los crowdworkers tenían que demostrar el uso de la batería Mac en sus vehículos de manera creativa. Los comerciales fueron subidos a una aplicación creada de manera especial para el concurso y el premio mayor consistía en \$ 5.000 dólares para el comercial ganador. Después de haber recibido 61 comerciales, un jurado calificó en vivo, mediante una twitcam, cada uno de los videos y eligieron a dos ganadores. El tercer lugar lo eligió la comunidad.

¿Qué logró la agencia de publicidad a través de este concurso y, a su vez, de la utilización de la técnica del Crowdsourcing? Generó a su cuenta un brand awareness (reconocimiento de la marca) y free press (divulgación en distintos medios) a nivel nacional, creó una comunidad de 10.000 fans y 61 comerciales de 30 segundos, que equivalen a $\$ 300.000$, que además fueron vistos por 18.000 personas.

\section{Presentación y discusión de los resultados de las encuestas}

La principal técnica de investigación aplicada en este estudio es de fuente primaria y de tipo descriptivo. El objetivo de la misma es conocer el nivel de difusión que el Crowdsourcing tiene en el medio publicitario ecuatoriano y definir creencias que los profesionales del ramo tienen al respecto.

Como población universo se tomó a las agencias de publicidad pertenecientes a la AEAP (Asociación Ecuatoriana de Agencias de Publicidad, 2015) y se complementó con la base de empresas del sector de Publicidad y Mercadeo con oficinas en Guayaquil, publicada por Ekos, guía de negocios en su portal (Ekos, s.f.). Al tratarse de un universo reducido, se realizó un censo del mismo. Para ello se planteó una encuesta a 45 profesionales del mundo publicitario y del diseño en la ciudad de Guayaquil, a través de una plataforma digital de recolección de datos, durante el mes de marzo de 2016. Los resultados obtenidos se presentan a continuación:

Gráfico 1. Para usted, Crowdsourcing es:

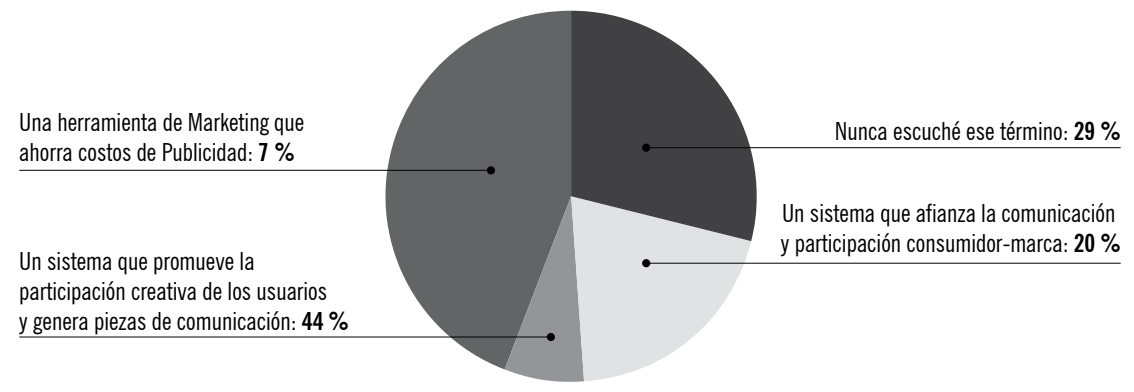

Fuente: Encuesta a profesionales de publicidad y diseño en Guayaquil

El primer interrogante pretende medir los distintos conceptos o ideas que los encuestados tienen sobre esta técnica. Se evidencia con claridad que existe cerca de un $30 \%$ de profesionales que no conoce del tema, pues nunca escuchó el término.
Sin embargo, la mayor parte de los usuarios relaciona la técnica con una participación creativa de no expertos o usuarios para generar piezas de comunicación para la marca. 
Gráfico 2. ¿Conoce de algún caso de éxito (local o internacional) donde la estrategia creativa principal se haya basado en Crowdsourcing?

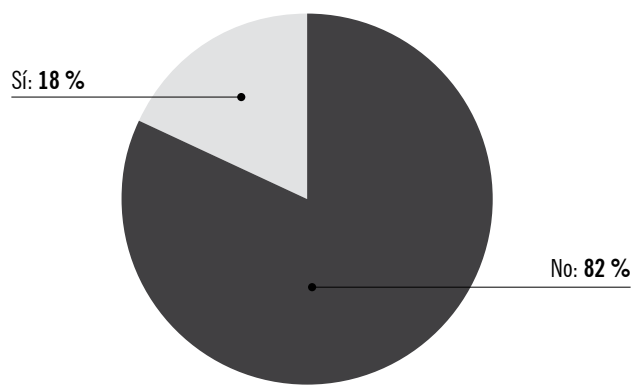

Fuente: Encuesta a profesionales de publicidad y diseño en Guayaquil

Pese a que la mayoría de los profesionales de de 45 encuestados. En su mayoría se refirieron al diseño y publicidad conocen algo de Crowdsourcing, caso Doritos, presentado en este estudio. También muy pocos han escuchado de algún caso concreto se mencionaron casos de empresas presentes en del uso exitoso de esta técnica. Ecuador como Baterías MAC, V220, Barcelona S.C.,

Las personas que mencionaron conocer algún y sugerencias de marcas como GoPro 0 Starbucks. caso exitoso de Crowdsourcing fueron apenas 8

Gráfico 3. En su opinión, la principal ventaja que el Crowdsourcing puede ofrecer al medio publicitario local es:

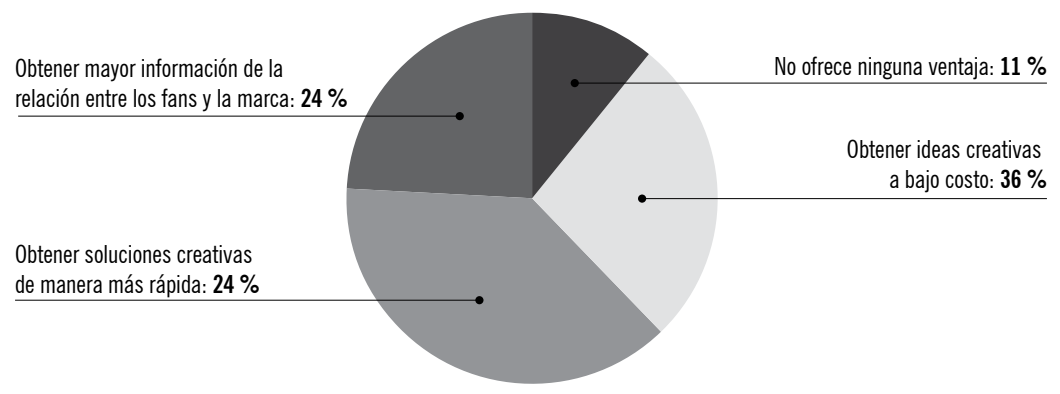

Fuente: Encuesta a profesionales de publicidad y diseño en Guayaquil

Los profesionales encuestados ven al Crowdsourcing como una técnica que les otorga alguna ventaja. Para la mayoría es una fuente para obtener información de la relación que tienen los consumidores leales y sus marcas. También hay quienes perciben a la técnica como una fuente de ideas creativas a bajo costo 0 de forma rápida. Tan sólo 5 personas consideran que no ofrece ningún beneficio. 
Gráfico 4. El Crowdsourcing podría darse con mayor frecuencia en el Ecuador:

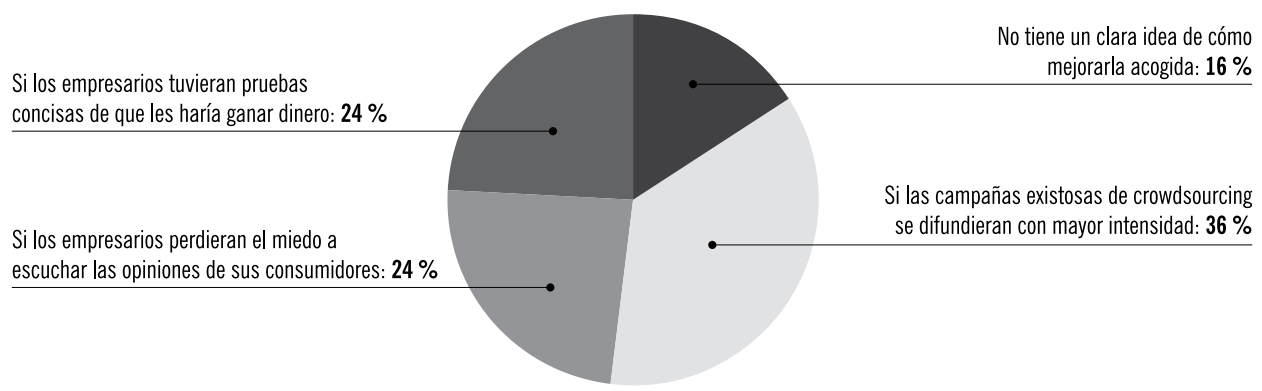

Fuente: Encuesta a profesionales de publicidad y diseño en Guayaquil

Una de los objetivos del estudio se enfoca en determinar cómo podría mejorarse la aplicación de esta técnica en el medio, puesto que en el estudio exploratorio ya se develó que existen muy pocas marcas que han recurrido a ella. En este sentido, el principal aspecto a mejorar, según los profesio- nales del ramo, es la difusión de los casos exitosos. Otro aspecto que ayudaría, según los encuestados, es proporcionar información más completa sobre el tema, de modo que los empresarios venzan el miedo a escuchar opiniones de sus consumidores.

Gráfico 5. ¿Podría recomendarnos algún website o plataforma para desarrollar acciones basadas en Crowdsourcing?

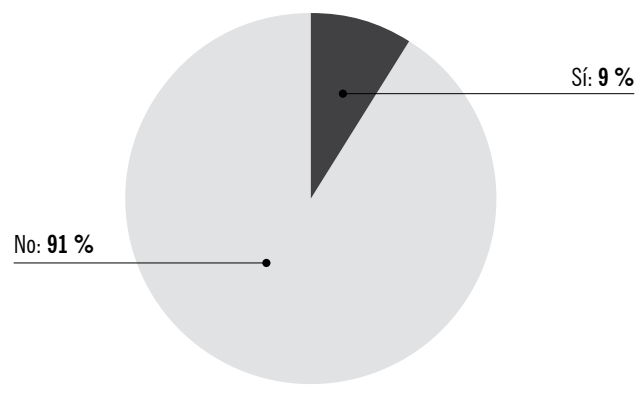

Fuente: Encuesta a profesionales de publicidad y diseño en Guayaquil

La última pregunta se refiere a las fuentes de referencia que los encuestados tienen como alternativa para consultar sobre el tema. Al ser todavía desconocido el Crowdsourcing, apenas cuatro personas remitieron algún sitio web. Dos de ellos mencionaron plataformas sociales como Youtube 0 Facebook, y dos citaron a http://www.infoCrowdsourcing.com/ y a http://www.Crowdsourcing.org/ 


\section{Conclusiones \\ y recomendaciones}

Según García (2011), gracias a la evolución tecnológica y la participación cada vez más activa de los consumidores en los distintos ámbitos de nuestra sociedad, estamos ante un cambio que ha descubierto la posibilidad de desarrollar proyectos colaborativos a gran escala.

El Crowdsourcing en poco tiempo se ha convertido en un modelo de producción y resolución de problemas a gran escala global, difundiendo los casos de estudio a personas totalmente desconocidas para la marca y que, a través de una convocatoria abierta, entran a formar parte del equipo de la empresa por un breve período.

Partiendo con un enfoque internacional, nos damos cuenta de que las empresas ya están utilizando la técnica y se está haciendo popular ya que, además de ser de gran ayuda para la generación de ideas, también reduce costos e impulsa a la colaboración masiva.

Aunque en este artículo se analiza la técnica desde la perspectiva de diversos autores, se puede constatar que globalmente no se ha llegado a un término concluyente del Crowdsourcing, dado que muchos de ellos tienen diferentes modos de verla. Unos lo ven como un modelo productivo, otros, como un modelo de negocio, y otros lo ven como una teoría. Pero se podría afirmar que todos tienen una base en común: la participación activa, la colaboración y la difusión de conocimientos compartidos.

El Crowdsourcing es una técnica eficiente como fuente de creatividad debido a que ayuda a resolver problemas y a cumplir con las metas trazadas. Esto se corrobora a través de los casos de estudio analizados. Concentrándonos en resultados, esta técnica crea valor y esto se traduce en resultados tangibles.

La visión de los profesionales de la publicidad y el diseño en el mercado ecuatoriano, especialmente de Guayaquil, determina el grado de difusión que la técnica ha logrado hasta ahora. La perspectiva del Crowdsourcing en nuestro medio ha sido vista de una manera muy superficial, pues la mayoría coincide en que en el Ecuador la técnica es conocida y utilizada como una herramienta que invita a los consumidores a generar ideas para una empresa 0 marca de forma voluntaria, pero sus resultados no se han promovido con la fuerza suficiente y son muy pocos los casos que se referencian.

La falta de información y de un correcto enfoque sobre el Crowdsourcing en las agencias de publicidad y las áreas de mercadeo hacen que la técnica no llegue a los niveles registrados en el mercado internacional.

Como un aporte, se puede anotar que los profesionales encuestados perciben que el Crowdsourcing les puede ofrecer una contribución significativa para la creatividad y la resolución de problemas de las marcas que manejan, y mencionan a la obtención de información, ideas creativas de bajo costo y rápida respuesta como las principales ventajas.

Por otra parte, dentro de las recomendaciones para que el Crowdsourcing se pueda desarrollar en cada uno de sus lugares de trabajo, se destaca el ampliar información de cómo funciona este esquema, de modo que los empresarios tengan mayor confianza en incorporar a sus consumidores en los procesos creativos y las propuestas de soluciones de comunicación.

Una vez realizado el análisis de los casos y de las encuestas, se puede afirmar que existen oportunidades en el mercado ecuatoriano, además de un cierto conocimiento de la técnica. Las interrogantes que se querían contestar en esta investigación se pueden resumir en un solo aspecto a recalcar: la difusión del correcto uso del Crowdsourcing a través de las campañas exitosas.

Por más que el Crowdsourcing se base esencialmente en la premisa de que «dos cabezas trabajan mejor que una», muchas veces la multitud puede generar información poco relevante y resulte una pérdida de tiempo. Por tanto, para resolver este problema, la técnica tiene que ser muy bien explicada, primero a los empresarios, para luego ser claramente enfocada a lo que se quiere lograr. 
Las empresas actualmente están dando un giro importante al considerar que no pueden cerrarse y aislarse ante un mundo tan cambiante, cuyas preferencias y gustos varían en períodos cada vez más reducidos. Sin embargo, en el medio publicitario ecuatoriano todavía no se dan pasos firmes respecto de la incorporación activa de los consumidores en los procesos creativos de la marca.
El Crowdsourcing, como técnica incipiente, irá madurando para encajar con lo que toda empresa busca. Desde luego, generará opiniones diversas que irán consolidándose con el tiempo, hasta develarse como una fuente de innovación y creatividad, que se recomienda ser ampliamente aprovechada.

\section{Referencias bibliográficas}

- Asociación Ecuatoriana de Agencias de Publicidad (AEAP). (2015). Asociación Ecuatoriana de Agencias de Publicidad. Recuperado en Marzo de 2016 de http://www.aeap.com.ec/.

- Azcarate, B.D. (2013). Comunidad ICEMD. Recuperado el 09 de marzo de 2015, de http://comunidad.ice$\mathrm{md} . c 0 \mathrm{~m} /$ comunicados/2013_14/ Newsletters/2013_09_06/index. html\#noticia9

- Brabham, D. (2008). Crowdsourcing as a Model for Problem Solving. Convergence: The International Journal of Research into New Media Technologies, 14(01), 75-90. D0I: 10.1177/1354856507084420.

- Effie Awards. (2008). Crash the superbowl. Recuperado el 02 de marzo de 2015 de http://s3.amazonaws.com/ effie_assets/2008/2592/2008_2592 pdf_1.pdf

- Ekos. (s.f.). Guía de negocios. Recuperado en marzo 2015 de http:// www.ekosnegocios.com/empresas/ resultados.aspx?ids $=278$
- El confidencial. (22 de octubre de 2011). Adtriboo, la democratización del talento. El confidencial. Recuperado el 13 de marzo de 2015 de http://www.elconfidencial.com/tecnologia/2011-10-22/adtriboo-la-democratizacion-del-talento_773343/ - Galmés, M. (2012). La base social como fuente de creatividad en contextos de crisis. Creativity and society, XVIII, 1-29. Recuperado en marzo de 2015 de http://www. creatividadysociedad.com/articulos/18/07_Crowdsourcing.\%20 La_base_social_como_fuente_ de creatividad.pdf

- Gómez, J., Grau, A., Ingellis, A., y Jabbaz, M. (2010). Técnicas cualitativas de Investigación Social. Valencia, España: Universidad de Valencia.

- Grover, A. (25 de Noviembre de 2006). Phantom Captain: Art and Crowdsourcing. Recuperado en Marzo de 2015 de http://www.andreagrover.com/phantom-captain/
- Howe, J. (01 de junio de 2006). The rise of crowdsourcing. Recuperado en Marzo de 2015 de Wired: https:// www.wired.com/2006/06/crowds/

- Kotler, P. (2014). 8 maneras de crecer. Madrid: LID Editorial Empresarial. - Martínez Carrasco, R.D. (2011). Investigación comercial: técnicas e instrumentos. España: Tébar.

- Martinez Rodríguez, J. (2011). Métodos de investigación cualitativa. Silogismos, 08(2), 1-34. Recuperado en marzo 2015 de http://www.cide. edu.co/doc/investigacion/3.\%20metodos\%20de\%20investigacion.pdf.

- Piller, F., y Walcher, D. (2006). To0Ikits for Idea Competitions: A Novel Method to integrate users in new product development. R\&D Management, 36(3), 307-318.

- Premios Iberoamericanos Social Media. (2013). Casos de exito. Recuperado el 30 de marzo de 2015 de Premios iberoamericanos Social Media: http://premiossm.com/premios-2013.html 
- Roig, A., Sanchez-Navarri, J., y Leibovitz, T. (2012). Crowdsourcing y crowdfonding como practicas colaborativas en la produccion audiovisual contemporénea. Esta pelicula la hacemos entre todos. Revista Icono 14, 10(1), 25-40.

- Shuttleworth, M. (16 de junio de 2010). Estudio transversal. Recuperado el 30 de marzo de 2015 de
Explorable: https://explorable.com/ es/estudio-transversal

- Shuurman, D., Baccarne, B., De Marez, L., y Mechant, P. (2012). Smart Ideas for Smart Cities: Investigating Crowdsourcing for Generating and Selecting Ideas for ICT Innovation in a City Context. Journal of Theoretical and Applied Electronic Commerce Research,
7(3), 49-62. D0I: 10.4067/S071818762012000300006.

- Whitla, P. (2009). Crowdsourcing and its application in marketing activites. Contemporary Management Research, 5(1), 15-28. Recuperado en marzo 2015 de http:// www.cmr-journal.org/article/viewFile/1145/264.

\section{Registro bibliográfico}

Barragán Castañeda, N., Santibáñez Vásquez, J.D., y Patiño Loor, A.C. (2016). Crowdsourcing como fuente de creatividad en el medio publicitario ecuatoriano. Revista Ciencias Económicas, 13(02) 157-169. 